\title{
LA IMIAGEN MATEMÁTICA DEL LENGUAJE: MÁS ALLÁ DE LA JERARQUIA DE CHOMSKY (LOS ECOSISTEMAS DE GRAMÁTICAS)
}

\author{
Carlos Martin Vide \\ (GRLMC, Universitat Rovira i Virgili)
}

\section{Contexto}

Una de las derivaciones del pensamiento cartesiano es lo que se ha venido en llamar "lingdistica cartesiana", paradigmáticamente representada en sus inicios por la gramática de Port-Royal. Ya a mediados de nuestro siglo, el pensamiento cartesiano sobre el lenguaje cristaliza en la concepción de Chomsky, que, entre otras cosas, concibe la gramática, y más concretamente la sintaxis, como teoria matemática de la estructura de la frase que se ocupa de caracterizar sistemas de generación. Asumiendo esa visión y el contexto mentalista del pensamiento cartesiano, Chomsky elabora su conocida jerarquia de lenguajes, que significó un hito fundamental en la investigación de las propiedades matemáticas de la sintaxis de las lenguas naturales y que constituye todavia hoy una referencia imprescindible en el análisis formal de las lenguas naturales. En sus primeros trabajos, Chomsky aúna mentalismo y matemáticas. Hoy, sin embargo, las cosas son bien distintas, porque la investigación matemática de la sintaxis de las lenguns naturales o de los lenguajes de programación ha conseguido adquirir un estatuto cientifico independiente dentro del marco de las ciencias de la computación.

La jerarquia de Chomsky ha resultado ser demasiado burda y gruesa para describir una lengua natural; también para otras finalidades en el ámbito de las ciencias de la computación. De ahi que se impusiera ya casi desde el principio la necesidad de diseiar sistemas de generación más ricos y ambiciosos. Inicialmente, el lingüista chomskyano trató de construir específicos mecanismos matemáticos de generación. Hoy, por contra, la situación es inversa: el lingüista debe, razonablemente, atender a interpretaciones lingüisticas plausibles de sistemas desarrollados fuera de su estricto ámbito, que además podrian llegar a tener alguna plausibjlidad cognitiva. De otra parte, parece ya insuficiente una caracterización estática de la lengua, siendo necesaria la consideración, más que del lenguaje como realidad primaria, de la interacción lingüistica como entidad dinámica en evolución.

¿Qué encontramos hoy por encima y más allá de la aludida jerarquia? Aceptemos que unos buenos parámetros para evaluar las virtudes de un mecanismo de generación pueden ser: su capacidad generativa (dentro de un cierto intervalo pequeî́o), su complejidad computacional (mínima) y su adecuación descriptiva (ajustada). Pues bien, hoy disponemos de toda una serie muy variada de mecanismos que ponen de manifiesto ventajas bien evidentes respecto a la jerarquia de Chomsky por lo que se refiere a aquellos parámetros: asi, por citar sólo unos pocos ejemplos, los sistemas de reescritura regulada, los sistemas de Lindenmayer, las gramáticas contextuales, los sistemas de gramáticas, las gramáticas dinámicas, los sistemas de computación molecular, etc., etc.

Como muestra de todos ellos, vamos a presentar de manera introductoria una clase de mecanismos generativos que podrian representar algunos fenómenos importantes de la realidad lingülística y cognitiva y que tienen la virtud de ser sugestivos metodológicamente: los ecosistemas de gramńticas. Mantengamos en mente que tales mecanismos son notablemente más tlexibles, potentes y computacionalmente cticientes que las gramáticas de Chomsky, a las que, sin embargo, la historia reserva un lugar destacado en su galeria de honor. 


\section{Ecosistemas de gramáticas}

\subsection{Presupuestos}

La noción de ecosistema de gramáticas está basada en una lista de postulados, a los que nos referiremos brevemente antes de definir formalmente el concepto:

(i) Un ecosistema consta de un entorno y unos agentes. El estado del entorno y los estados de los agentes se describen mediante cadenas de simbolos tomados de ciertos alfabetos.

(ii) Existe un reloj universal, que marca unidades de tiempo, las mismas para los agentes y para el entorno, y de acuerdo con el cual tiene lugar la evolución de aquéllos y de éste.

(iii) Tanto el entorno como los agentes tienen reglas de evolución características, que son reglas de sistemas de Lindenmayer que se aplican de un modo paralelo a todos los símbolos que describen los estados del entorno y de los agentes. Cada paso de la evolución tiene lugar en una unidad de tiempo.

(iv) Las reglas de evolución del entorno son independientes de los agentes y del estado del entomo. Las reglas de evolución de los agentes dependen del estado del entomo, de tal manera que en cada momento se escoge un subconjunto de reglas aplicables del conjunto general de cada agente.

(v) Los agentes actuan sobre el entorno de acuerdo con reglas de acción, que son reglas de reescritura de tipo secuencial. En cada unidad de tiempo, cada agente emplea una regla de acción, que elige de un conjunto que depende del estado actual del agente.

(vi) La acción de los agentes sobre el entorno tiene prioridad sobre la evolución de éste. Así, en una unidad de tiempo las reglas de evolución del entorno reescriben de manera paralela sólo aquellos simbolos de éste no afectados por la acción de los agentes.

\subsection{Estructurn}

Definición I. Un ecosistema de gramáticas es una (n+1)-upla:

$$
\Sigma=\left(E, A_{1}, \ldots, A_{n}\right)
$$

En ella $E=\left(V_{E}, P_{E}\right)$, donde $V_{E}$ es un alfabeto finito y $P_{E}$ es un conjunto finito de reglas de Lindenmayer de tipo 0 (en adelante, $O L$ ) sobre $V_{E}, y$ :

$$
A_{i}=\left(V_{i}, P_{i}, R_{i}, \phi_{i}, \psi_{j}\right)
$$

para $\mathrm{l} \leq \mathrm{i} \leq \mathrm{n}$, donde $V_{\mathrm{i}}$ es un alfabeto finito, $P_{\mathrm{i}}$ es un conjunto finito de reglas de tipo $0 \mathrm{~L}$ sobre $V_{i}, R_{i}$ es un conjunto finito de reglas de la forma $x \otimes y\left(\operatorname{con} x \in V_{E^{+}}, y \in V_{E}^{*}\right) y:$

$$
\begin{gathered}
\phi_{i}: V_{E} \otimes \wp\left(P_{i}\right), \\
\psi_{i}: V_{i}+\circledast \wp\left(R_{i}\right)
\end{gathered}
$$

son funciones, con $1 \leq \mathrm{i} \leq \mathrm{n}$. E es el entorno, con su alfabeto $\mathrm{V}_{\mathrm{E}}$ y su conjunto de reglas de evolución $\mathrm{P}_{\mathrm{E}}$ A su vez, $\mathrm{A}_{\mathrm{i}}$ es el agente $\mathrm{i}$, con su alfabeto $\mathrm{V}_{\mathrm{i}}$, su conjunto de reglas de evolución $\mathrm{P}_{\mathrm{i}}$ y su conjunto de reglas de acción $\mathrm{R}_{\mathrm{i}}$. Con $\phi_{\mathrm{j}}$, dependiendo del estado del entorno, seleccionamos las reglas de la evolución actual de $A_{i}$, y $\operatorname{con} \psi_{i}$, dependiendo del estado actual de $A_{i}$, escogemos las reglas de la acción actual de $A_{i}$. 


\subsection{Estados}

Un ecosistema de gramáticas funciona cambiando de estado, esto es, modificando las cadenas que representan los agentes y el entorno en un momento determinado.

Definición 2. Un estado de un ecosistema de gramáticas $\Sigma=\left(E, A_{1}, \ldots, A_{n}\right)$ es una $(n+1)$-upla:

$$
\sigma=\left(w_{E}, w v_{1}, \ldots, w_{n}\right)
$$

donde $w_{E} \in V_{E}^{*}, w_{i} \in V_{i}^{*}$, para $1 \leq i \leq n$. Asi, $w_{E}$ es el estado del entorno y $w_{i}$ el estado del agente i. El cambio del estado actual del sistema es el resultado de la intervención conjunta de las acciones y las evoluciones.

Definición 3. Sea $\sigma=\left(w_{E}, w_{1}, \ldots, w_{n}\right)$ un estado de un ecosistema de gramáticas $\Sigma=\left(E, A_{1}, \ldots, A_{n}\right)$. Decimos que $A_{\mathrm{i}}$ está activo en $\sigma$ si $\psi_{\mathrm{i}}\left(w_{\mathrm{i}}\right) \neq \Theta$, o sea, si el conjunto de sus actuales reglas de acción no es vacio. Una acción de un agente $A_{\mathrm{j}}$ activo en el estado $\sigma$ es una aplicación de una regla actual de acción $\mathrm{r} \in \psi_{\mathrm{i}}\left(\mathrm{w}_{\mathrm{i}}\right)$ en el estado del entomo $\mathrm{w}_{\mathrm{E}}$. Una acción simultánea de agentes $A i_{1}, \ldots, A i_{r}$, con $\left\{i_{1}, \ldots, i_{r}\right\} \in\{1, \ldots, n\}$, activos en $\sigma$ sobre el entomo es un paso en una derivación paralela $w_{E} \Rightarrow w_{E}^{\prime}$ tal que:

$$
\begin{aligned}
& w_{E}=x_{1} u_{1} x_{2} u_{2} \ldots u_{t} x_{r+1}, \\
& w_{E}=x_{1} v_{1} x_{2} v_{2} \ldots v_{r} x_{r+1},
\end{aligned}
$$

donde:

$$
\begin{gathered}
u_{j} \otimes v_{j} \in \psi_{i j}\left(w_{i j}\right), \text { con } l \leq j \leq r, y \\
x_{i} \in V_{E}^{*}, \text { con } l \leq i \leq r+l .
\end{gathered}
$$

Definición 4. Sea $\sigma=\left(w_{E}, w_{1}, \ldots, w_{n}\right)$ un estado de un ecosistema de gramáticas $\Sigma=$ $\left(E, \Lambda_{1}, \ldots, A_{n}\right)$. Decimos que $w_{i}$ ' es una evolución actual de un agente $A_{j}$ en un estado $v_{j}$ si $w_{i}$ ' se puede derivar de $w_{i}$ mediante producciones de $\phi_{i}\left(w_{E}\right)$, con $\mathrm{l} \leq \mathrm{i} \leq \pi$, en el modo $0 \mathrm{~L}$. Decimos que un estado $w_{E}$ 'es una evolución del entorno a partir del estado $w_{E}$ si $w_{E}$ ' se puede derivar de $\mathrm{w}_{\mathrm{E}}$ mediante producciones de $\mathrm{P}_{\mathrm{E}}$ en el modo $0 \mathrm{~L}$. Un cambio de estado de un ecosistema de gramáticas es una evolución de los estados de todos los agentes y una evolución del entorno en todos los puntos excepto en aquellos en los que los agentes actualmente activos estản desarrollando simultảneamente una acción.

Definición 5. Sean $\sigma=\left(w_{E}, w_{1}, \ldots, w_{11}\right), \sigma^{\prime}=\left(w_{E}, w_{1}, \ldots, w_{11}\right)$ dos estados de un ecosistema de gramáticas $\Sigma=\left(E, A_{1}, \ldots, \wedge_{n}\right)$. Decimos que $\sigma$ cambia a $\sigma^{\prime}$ (o $\sigma^{\prime}$ se deriva directamente de $\left.\sigma\right)$, y lo escribimos $\sigma \Rightarrow_{\Sigma} \sigma^{*}$, si se cumplen las dos siguientes condiciones:

(i) $w_{E}$ ' se obtiene de $w_{E}$ a través de una evolución producida por todos los agentes activos en el estado $\sigma$, es decir, cuando:

$$
\begin{gathered}
w_{E}=z_{1} x_{1} z_{2} x_{2} . . z_{1 m} x_{m} z_{m+1}, \\
w_{E}^{\prime}=z_{1} y_{1} z_{2} y_{2} . . z_{m} y_{m} y_{m+1},
\end{gathered}
$$

tales que: 
a) $z_{1} x_{1} z_{2} x_{2} . . z_{m n} x_{m} z_{m+1} \Rightarrow_{\Sigma} z_{1} y_{1} z_{2} y_{2} \ldots z_{m l} y_{m} z_{m+1}$ es una acción simultánea de todos los agentes $A i_{1}, \ldots, A i_{m}$, con $\left\{i_{l}, \ldots, i_{m}\right\} \subseteq\{1, \ldots, n\}$, que están activos en $\sigma, y$

b) $z_{1}{ }^{\prime} z_{2}$ '... $z_{m}{ }^{\prime} z_{n+1}$ 'es una evolución de $z_{1} z_{2} \ldots z_{m} z_{m+1} ; y$

(ii) $w_{i}$ ' es una evolución actual de $A_{i}$ en el estado $w_{i}$, con $1 \leq i \leq n$.

La clausura reflexiva y transitiva de $\Rightarrow_{\Sigma}$ se denota con $\Rightarrow_{\Sigma}{ }^{*}$.

De esta forma, el siguiente estado del agente está únicamente determinado por sus reglas de evolución, mientras que el siguiente estado del entorno depende tanto de sus propias reglas de evolución como de las interacciones que mantiene con los agentes actualmente activos. $\mathrm{Si}$ un agente llega al estado vacio, se queda inactivo durante el funcionamicnto restante del sistema. (Obsérvese que el dominio de la función $\psi_{\mathrm{i}}$ en la definición de ecosistema de gramáticas es $V_{i}{ }^{+}$.) Algo distinto sucede cuando el agente no selecciona ninguna de sus reglas de acción, caso en el que el agente queda sólo temporalmente inactivo.

\subsection{Secuencia de estados}

Las secuencias de estados caractcrizan el comportamiento evolutivo del ecosistema. Como dichos estados, tanto de los agentes como del entomo, pueden expresarse como cadenas, podemos considerar un ecosistema como un mecanismo de generación que asocia ciertos lenguajes a un estado inicial.

Definición 6. Sea $\Sigma$ un ecosistema y sea $\sigma_{0}$ un estado inicial de $\Sigma$. El conjunto de secuencias de estados de $\Sigma$ es:

$$
\operatorname{Seq}\left(\Sigma, \sigma_{0}\right)=\left\{\left\{\sigma_{i}\right\}_{i=0}{ }^{x} \mid \sigma_{0} \Rightarrow_{\Sigma} \sigma_{1} \Rightarrow_{\Sigma} \sigma_{2} \Rightarrow_{\Sigma} \cdots\right\}
$$

$\operatorname{Seq}\left(\Sigma, \sigma_{0}\right)$ indica el comportamiento del ecosistema, en términos del conjunto de secuencias posibles de estados a partir del estado inicial.

El conjunto de secuencias de estados del entorno es:

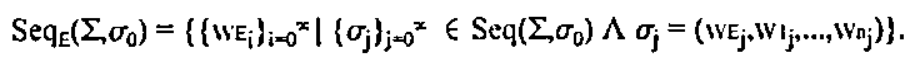

El conjunto de secuencias de estados del agente $\mathrm{A}_{\mathrm{j}} \mathrm{es:}$

$$
\operatorname{Seq}_{j}\left(\Sigma, \sigma_{0}\right)=\left\{\left\{w_{j k}\right\}_{k=0} x \mid\left\{\sigma_{k}\right\}_{k=0} x \in \operatorname{Seq}\left(\Sigma, \sigma_{0}\right) \wedge \sigma_{k}=\left(w_{E_{k}}, w_{i_{k}}, \ldots, w_{j k}, \ldots, W_{\pi_{k}}\right)\right\} .
$$

El lenguaje del entorno es:

$$
L_{E}\left(\sum, \sigma_{0}\right)=\left\{w_{E} \in V_{E}^{*} \mid\left\{\sigma_{j}\right\}_{j=0} x \in \operatorname{Seq}\left(\sum, \sigma_{0}\right) \wedge \sigma_{j}=\left(w_{E}, w_{1}, \ldots, w_{n}\right)\right\}
$$

El lenguaje del agente $A_{\mathrm{i}}$ es:

$$
\left.L_{i}\left(\sum \sigma_{0}\right)=\left\{w_{j} \in V_{i}^{*} \mid\left\{\sigma_{j}\right\}_{j=0} x \in \operatorname{Seq} \sum \sigma_{0}\right) \wedge \sigma_{j}=\left(w_{E}, w_{1}, \ldots, w_{i}, \ldots, w_{n}\right)\right\},
$$

para $i=1,2, \ldots, n$. 
Ejemplo 7. Veamos cómo incluso un solo agente puede producir un cambio significativo en la evolución del entorno. Para ello, sea $\Sigma=\left(E, A_{1}\right)$ un ecosistema de gramáticas en el que:

$$
\begin{gathered}
E=\left(V_{E}, P_{E}\right), \\
V_{E}=\{a\}, \\
P_{E}=\{a \otimes a\}, \\
A_{1}=\left(V_{1}, P_{1}, R_{1}, \phi_{1}, \psi_{1}\right), \\
V_{1}=\{b\}, \\
P_{1}=\{b \otimes b\}, \\
R_{1}=\left\{a \otimes a^{4}\right\}, \\
\phi_{1}(v)=P_{1},-w \in V_{E}{ }^{*}, \\
\psi_{1}(u)=R_{1},-u \in V_{1}+.
\end{gathered}
$$

Sea $\sigma_{0}=(a, b)$ el estado inicial. En estas condiciones:

$$
\operatorname{Seq}\left(\sum, \sigma_{0}\right)=\left\{(a, b),\left(a^{4}, b\right),\left(a^{7}, b\right), \ldots,\left(a^{3 i+1}, b\right), \ldots\right\}
$$

y:

$$
\begin{gathered}
\mathrm{L}_{\mathrm{E}}\left(\sum, \sigma_{0}\right)=\left\{\mathrm{a}^{3 \mathrm{i}+1} \mid \mathrm{i} \geq 0\right\}, \\
\mathrm{L}_{1}\left(\Sigma, \sigma_{0}\right)=\{\mathrm{b}\} .
\end{gathered}
$$

Asi pues, la interacción entre dos sistemas $0 \mathrm{~L}$ muy simples, el del agente y el del entorno, da como resultado un lenguaje infinito que no es un lenguaje $0 \mathrm{~L}$.

Si sustituimos $P_{1}$ por $P_{1}=\{b \otimes \lambda\}$, entonces el agente, después del primer paso, no actúa sobre el entorno, $y$ :

$$
\begin{gathered}
\operatorname{Seg}\left(\Sigma, \sigma_{0}\right)=\left\{(\mathrm{a}, \mathrm{b}),\left(\mathrm{a}^{4}, \lambda\right)\right\}, \\
\mathrm{L}_{\mathrm{E}}\left(\Sigma, \sigma_{0}\right)=\left\{\mathrm{a}, \mathrm{a}^{4}\right\}
\end{gathered}
$$

Este tampoco es un lenguaje 0L.

Ejemplo 8. Veamos ahora que también el entorno puede producir cambios esenciales en el comportamiento del agente. Sea ${ }^{\mathrm{M}}=(\mathrm{E}, \mathrm{Al})$ un ecosistema de gramáticas, en el que:

$$
\begin{gathered}
E=\left(V_{E}, P_{E}\right), \\
V_{E}=\{e, f\}, \\
P_{E}=\{e \otimes e, f \otimes f\}, \\
A_{1}=\left(V_{1}, P_{1}, R_{1}, \phi_{1}, \psi_{1}\right), \\
V_{1}=\{a\}, \\
P_{1}=\left\{a \otimes a, a \otimes a^{3}\right\}, \\
\phi_{1},(c)=\left\{a \otimes a^{3}\right\}, \\
\phi_{1}(f)=\{a \otimes a\}, \\
R_{1}=\{e \otimes f, f \otimes f\}, \\
\psi_{1}(a)=\{e \otimes f\}, \\
\psi_{1}(a)=\{f \otimes f\} .
\end{gathered}
$$


En estas condiciones:

$$
L_{1}(\Sigma,(e, a))=\left\{a, a^{3}\right\}
$$

que tampoco es un lenguaje $0 \mathrm{~L}$, con lo que se ve modificado el comportamiento del agente por influencia del entorno.

\section{BIBLIOGRAFÍA}

CSUHAJ-VARJÚ, E., J. DASSOW, J. KELEMEN \& GH. PAUN (1994), Grammar systemis. A grammatical approach to distribution and cooperation. Gordon and Breach, London.

DASSOW, J. \& GH. PAUN (1989), Regulated rewriting in formal language theory. Springer, Berlin.

KARHUMĀKI, J., H. MAURER \& G. ROZENBERG, eds. (1994), Important results and trends in theoretical computer science. Springer, Berlin.

PAUN, GH., ed. (1994), Mathematical aspects of natural and formal languages. World Scientific, Singapore.

PAUN, GH., ed. (1995), Artificial life: grammatical models. Black Sea University Press, Bucharest.

ROZENBERG, G. \& A. SALOMAA (1980), The mathematical theory of $L$ systems. Academic Press, New York.

SALOMAA, A. (1973), Formal languages. Academic Press, New York. 